diverticulum. The patient did very well. The chief point of interest is the fact that the opening from the diverticulum into the bowel was large enough for one's little flnger to pass through, so that it must have been simple stasis of the contents that started the inflammation; there was no kinking. The patient made an $\epsilon$ xcellent recovery.

Case 17.

Female, aged 35, She gave a long history of attacks of abdominal colic accompanied by vomiting. When I flrst saw her it was for a strangulated right femoral hernia. She had had a lump there some time, and I hoped that that had been the cause of her frequent attacks of colic. She was much better for a fortnight after the operation but she then began to get pain in her abdomen, followed in a few days by a very bad attack of colic and signs of intestinal obstruction. I saw her again, and there was no doubt about the obstruction. It was not complete, as I could feel the intestinal contents being squeezed through the constriction. I advised operation, but as she felt better that day it was refused. She got worse, however, and I opened the abdomen and found a very tight stricture of the small intestine. It was very difficult to get at, as it was fixed so firmly to the abdominal wall that the adhesions had to be cut with scissors. They were like cartilage. I excised the stricture, and did an end-to-end anastomosis. She got quite well, and never had any more attacks of colic. The stricture would only allow the passage of a No. 9 catheter.

Case 18.

Male, aged 75. He had not been well for a week; he had had an irreducible hernia on the right side for some years. About a week ago he had pain in abdomen and vomiting. This forced something more down into his hernia, and made it painful. Bowels opened well until I saw him. Has vomited off and on since the attack began. On the right side he had a large irreducible hernia, not tense, and only tender at the top. I operated at once. The hernia sac was a very large one, contained a quantity of fluid, a large amount of small intestine very adherent to the sac, and at the upper part some thickened and matted omentum adherent to the sac and to intestine. On further examination of this, one part of it was found to be wrapped round an inflamed appendix, a few drops of pus escaping while exposing it. The wound had to be extended, so as to open the general peritoneal cavity in order to get at the caecum for the purpose of removing the appendix. Patient made perfect recovery.

Case 10.

Male, aged 10. Four days before I saw him he was taken with acute pain in the abdomen and vomiting. Two days later a lump was noticed in the right side of the scrotum. This swelling increased and became very painful. Bowels acted the day before I saw him. The boy looked extremely ill. There was a right-sided scrotal swelling, tender, red. There was pain and tenderness just above internal ring; nothing felt there. Was diagnosed as a probable torsion of the cord. An incision was made over the swelling, and on opening what was taken to be the tunica, a quantity of very foul pus was evacuated. As the pus was coming from above, the incision was extended up to expose the general peritoneal cavity. At the mouth of the hernia sac was found a perforated appendix, surrounded by an abscess which had evidently burst into the hernia sac. There was also a necrotic area on the caecum. The boy did not recover from the shock of the operation, and died the next day.

\section{SUGGESTIONS FOR THE TECHNIQUE AND PERFORMANCE BY A NEW METHOD OF WERTHEIM'S ABDOMINAL PANHYSTERECTOMY.*}

By CHARLES P. CHILDE, F.R.C.S., SENIOR SURGEON, ROYAL PORTSMOUTH HOSPITAL.

Two dangers are conspicuous in the performance of Wertheim's abdominal panhysterectomy for cancershock and sepsis-shock because the operation under the most favourable conditions is a severe and prolonged one; sepsis because in addition to circumstances con ducive to infection being present, a septic stage is intro-

* A paper read before the Gynaecological Section of the Royal Society of Medicine, Ju.y 3rd, 1913. duced of necessity into the operation itself. It will be understood that I am omitting the normal risk of infection which attends the performance of every operation, and which can be practically eliminated by a careful operative technique.

The following are the special circumstances favourable to infection in Wertheim's operation :

1. Patients suffering from the disease for which the operation is performed are usually below the average standard of health, their resistance power against infection is diminished, and they respond, therefore, readily to it

2. In addition to the peritoneal cavity, two extensive wounds are exposed to infection during the operation-one in the abdominal wall, the other in the pelvis. The former, moreover, is one whose surfaces have been a good deal pulled upon and contused in the retraction necessary for the performance of the operation, and wound surfaces injured in this manner offer a poor resistance to infection.

3. The pelvic wound is open to the external surface by way of the vaginal canal.

4. Retzius's space is freely opened, and this dead space offers a favourable nidus for the accumulation of wound discharges and their subsequent infection.

Furthermore the patient may be actually infected at the time of operation from a foul ulcerating growth, though in operable cases there is as a rule no evidence of this as judged by the pulse and temperature.

When it is considered that in the presence of the above mentioned favourable conditions for infection we of necessity introduce a septic stage as part of the operation, it is not surprising that infection should readily occur. Most of the conditions favouring infection are inherent in the operation itself and cannot be avoided. An important exception in this connexion, and one which Wertheim has insisted upon, is the method of retraction of the abdominal wound. Gloved fingers alone should be em. ployed in this manœuvre, as they are the least harmful retractors, and bruise the wound surfaces much less than heavy metal ones. The matters over which we possess chief control in lessening the risk of infection are the diseased and septic uterine cavity and tho pelvic wound left after removal of the uterus, and according to the manner in which these are managed will $d \in$ pend mainly the results obtained. In the manage. ment of the septic uterine cavity the following is my own practice. To begin with, I do not think that preliminary treatment is efficacious. Various preliminary measures, such as antiseptic vaginal douching, etc., may be adopted according to the fancy of the operator, but disinfection to be efficacious must be done as part of the operation itself and after the patient has been anaesthetized. Formerly, after thoroughly curetting away the soft growth a few days before operation, and applying carbolic acid or iodized phenol to the surface, $I$ used to employ in the interval copious antiseptic vaginal donching followed by a thorough cleansing of the vagina by the house-surgeon, with the patient in the lithotomy position, just before she was brougint to the theatre. This was in order to lessen the time the patient was under the anaesthetic. I was never, however, satisfied with the results obtained even under the most careful régime. Frequently a little dis. charge was seen on dividing the vagina; frequently in fection of the abdominal or pelvic wound occurred. I have consequently for some time past abandoned this method in favour of the following, notwithstanding the extra ten minutes or quarter of an hour it adds to the operation, and it has yielded far more satisfactory results. In fact, infection lias disappeared, though formerly it was as high as 30 per cent. The patient, anaesthetized for the operation and just previous to it, is placed in the lithotomy position. All the soft growth is removed with scissors and sharp spoon. The raw surface left is very thoroughly cauterized with Paquelin's cautery. The vagina is then scrupulously dried and painted with 2 per cent. iodine in spirit. Finally, the raw surface and the vagina are closely packed with dry sterile gauze, one end of which is left hanging out of the vagina. When the vagina is about to be divided the gauze is withdrawn by an assistant not taking part in the operation. If the above procedure is efficiently carried out, the vagina on division is, as it should be, perfectly dry. Wertheim 
himself dries the vagina out immediately before its division with sterile swabs on long-handled forceps. This not only necessitates the surgeon desisting from the or eration for a few valuable minutes, but it is not so certain of securing an absolutely dry vagina, and is objectionable owing to the operator taking part in an $\mathrm{n}$.ecessary septic procedure during the performance of the operation. The area about the uterus is surrounded with two large gauze packs soaked in salt solution just previous to division of the vagina, and immediately after its division these are folded round so as to enclose the uterus while it is lifted out of the pelvis.

An equally important consideration is the technique to bə adopted for the pelvic wound; the most important points here are :

1. Securing perfect haemostasis if possible.

2. Leaving no foreign bodies, such as ligatures, in the wound, of which I shall speak directly.

3. Discarding of gauze for drainage purposes.

Where I have secured perfect haemostasis - that is, in ncarly all cases - I employ no drainage whatever. The only cases in which gauze is employed are the quite exceptional ones in which venous oozing is impossible to arrest, at all events without much loss of time. Such cases have occurred to me. In these I have employed gauze for haemostatic but not for drainage purposes, and it is removed in twenty-four hours. Formerly following the practice recommended by Wertheim, and, I believe, still adopted by him and others, I used to leave gauze packing in the pelvic wound for five or six days. On withdrawal it was invariably exceedingly foul, and I cannot believe, notwithstanding Wertheim's high authority for it, that it is a sound practice to follow.

The second conspicuous danger in Wertheim's operation is shock or exhaustion. For this, again, there are favouring conditions-for example, the low state of nutrition owing to loss of appetite, wearing pain, and frequent losses of blood and discharge from the ulcerating growth. These we have to talie generally as we find them. We are not dealing with a disease in which we can afford to spend much time in building the patient up. At the same time in inoperable cases patients will improve so strikingly for a short time from the simple operation of curetting away the soft growth, and so arresting the discharges, that in some operable cases, where the general condition is unfavourable owing to constant losses, and in which it is doubtful whether the patient will stand the operation, this step should, I think, be resorted to as a preliminary measure, and the operation may with advantage be postponed for two or three weeks, when the patient will frequently be found to be in a much better condition to go through it. : Usually, however, the operation is performed without any delay, and inasmuch as even in the casiest cases it is a severe and prolonged one, any steps are desirable which facilitate it and make for the saving of time, provided that they are surgically sound. Formerly finding a ureter, especially the left ureter in a fat subject, was sometimes a difficulty to me and a cause of delay, but, if the ureters are not exposed at once on opening up the broad ligament, they can always be readily felt between the forefinger and thumb, and no time need therefore be wasted over this step.

In my experience the most prolonged part of the operation is dealing with the parametrium and vagina-that is, applying the ligatures to the sections of parametrium in a fat subject, or one with a deep pelvis, or where the uterus is fixed and cannot be drawn up, and stitching round the vaginal wall after it has been divided. Now, there is a quicker, and, I believe, a better, way of dealing with these steps of the operation, and latterly I have only employed four ligatures in the operation-one for each ovarian and one for each uterine artery (of silk). The round ligament contains no large ressel, and is crushed and dicided without ligature. The parametrium, after the uterine arteries have been tied, contains no large vessel. It is clamped in sections with strong crushing clamps, divided, and the divided surfaces are seared with Paquelin's cautery before the clamps are removed. Haemorrhage from the vaginal wall is easily arrested by applying Wertheim's vaginal fcrseps, furnished with augmented crushing power, to the portion of the vagina to be divided for a moment beforo division. This, after division, is also seared with Paquelin's cautery. By these measures perfect haemostasis is secured, and the pelvic wound is perfectly dry. Additional advantages of this method are that the cauterizing of the parametrium will destroy any cancer cells which may be present in the seared portion and that no ligatures which may act as sources of irriti. tion or infection are left in the pelvic wound. Wertheim's forceps, which are only intended for securing these struc. tures preparatory to ligaturing them, have little crushing power, and are unsuitable. The instruments, which I have

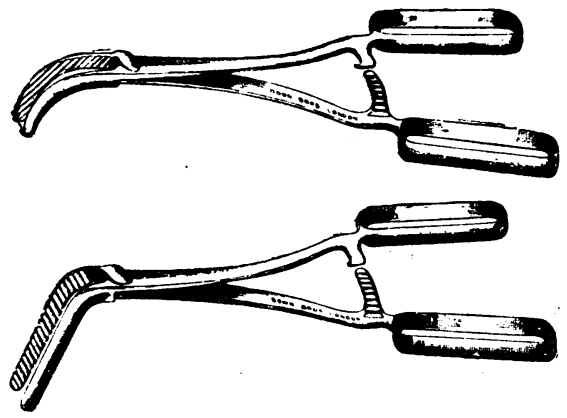

had made for me by Down Bros., possess sufficient crush. ing power for the purpose. They are also made with shorter handles, which, while quite long enough for any case, render them much more handy to apply. The peritoneum is finally sewn over, and the abdominal wounct is closed in layers. If any bleeding occurs, it is from veins opened in separating off the bladder at the side of the cervix, and a ligature or two may be required here; if it takes the form of general oozing, with no vessel to ligature, adrenalin will sometimes arrest it; otherwise some gauze packed into the bleeding area, with an end thrust through the vagina, suffices. This, as I have said, is removed in twenty-four hours. I have adopted the method above described in 10 consecutive cases. In none has there been any bleeding. I submit it as an easier, quicker, and better way of performing this part of the operation.

\section{A CASE OF}

CARCINOMA OF THE PELVIC COLON, OVARIAN TUMOUR, AND APPENDICITIS, NECESSITATING REPEATED ABDOMINAL SECTION.

BY LAWRIE McGAVIN, F.R.C.S.ENG.,

SURGEON TO THE DREADNOUGHT HOSPITAL AND TO THE HOSPITAL FOR WOMEN ; LECTURER ON CLINICAL SURGERY IN THE IONDON (POST-GRADUATE) SCHOOL OF CLINICAL MEDICINE.

THE case here recorded is, I think, of interest from several points of view. First, the patient has survived the removal of an intestinal cancer for a period of over four and a half years without recurrence; secondly, she has undergone successfully the operations of oöphorcetomy (twice), colectomy, lateral implantation, appendicectomy, and the reposition of a colostomy combined with a proctopiasty-in all five laparotomies, besides having had a naevoid growth of her bladder cauterized; thirdly, although completely thrown out of action for so long a period, the rectum and sphincters took on their work at once on being again called into play.

\section{History of the Case.}

B. C., a married woman of the age of 38, was admitted to hospital for pain in the left side of the abdomen, in September, 1908. The pain was especially bad at her periods, and there was tenderness at a point just internal to the anterior iliac spine, and in the left vaginal fornix. She suffered from gradually increasing constipation. Her condition appears to have been áiagnosed as chronic ovaritis, and her abdomen was opened by a well-known gynaecologist. The left ovary and tube were found adherent to a mass in the lower part of the pelvic colon, but were not in themselves diseased. They were, how. ever, removed, and the mass in the sigmoid was either ignored, overlooked, or considered inoperable, and the 